\title{
Growth, optical, and electron transport studies across isotype $n$-GaAs/n-Ge heterojunctions
}

\author{
Mantu Kumar Hudait ${ }^{\text {a) }}$ \\ Materials Research Centre, Indian Institute of Science, Bangalore 560 012, India \\ and Central Research Laboratory, Bharat Electronics, Bangalore 560 013, India \\ S. B. Krupanidhib) \\ Materials Research Centre, Indian Institute of Science, Bangalore 560 012, India
}

\begin{abstract}
A diode structure consisting of a polar epilayer on a nonpolar substrate grown by metalorganic vapor phase epitaxy often faces problems of antiphase domain formation in the polar semiconductor and cross diffusion across the heterointerface. Ge outdiffusion into GaAs epilayers was studied by low temperature photoluminescence spectroscopy after etching the film from the surface. The absence of $p$ - $n$ junction formation inside the Ge substrate from interdiffusion of $\mathrm{Ga}$ and As has been studied by current-voltage characteristics using mesa diodes. These observations were confirmed by electrochemical capacitance voltage polaron profiler and secondary ion mass spectroscopy techniques. To understand the material quality and current conduction mechanism across the GaAs/ Ge heterojunction, $I-V$ characteristics of Si-doped $n$-GaAs $/ n-\mathrm{Ge}$ isotype heterojunctions using $\mathrm{Au}$ Schottky diodes have been studied for different doping densities. A plethora of growth conditions appear in the literature concerning the attempt to grow antiphase domain (APD)-free GaAs on Ge. In the present case, even though the growth temperature regime is close to reported values, the main difference in minimizing APD formation may arise from the growth rates $(\sim 3 \mu \mathrm{m} / \mathrm{h})$ and the V/III ratio $(\sim 88)$.
\end{abstract}

\section{INTRODUCTION}

GaAs/Ge epitaxial heterostructures (HSs) have received a great deal of attention as starting materials for the fabrication of space quality solar cells ${ }^{1-6}$ and their potential application in electronic and optoelectronic devices. ${ }^{7-9}$ Due to its high mechanical strength, $\mathrm{Ge}$ is an optimized substrate material in terms of its power-to-weight ratio for high efficiency GaAs/Ge photovoltaic devices. ${ }^{10,11}$ As large area, minority carrier devices, III-V/Ge cells are extremely sensitive to defects. The elimination of antiphase domains (APDs), which are characteristic of polar-on-nonpolar epitaxy, and suppression of large-scale interdiffusion across the GaAs/Ge heterointerface remain key challenges for increased yield, reliability, and performance.

GaAs on Ge, i.e., polar-on-nonpolar epitaxy by metalorganic vapor phase epitaxy (MOVPE) is an important issue regarding the optical and electrical properties not only from a fundamental point of view but also for device applications, such as buffer and base layers of solar cells. A polar-onnonpolar heterojunction diode presents two problems, namely, APD formation in the epitaxial polar semiconductor and cross diffusion at the heterointerface, which leads to uncontrolled doping on both sides of the junction. It has been shown that APD formation in GaAs on Ge can be suppressed through use of a vicinal (100) substrate miscut several degrees towards $[011] .{ }^{12-14} \mathrm{~A}$ number of reports in the literature examine cross diffusion at the GaAs-Ge

${ }^{a)}$ Electronic mail: mantu@mrc.iisc.ernet.in

b) Author to whom correspondence should be addressed; electronic mail: sbk@mrc.iisc.ernet.in heterointerface, ${ }^{14-17}$ yet there is little reported on the electrical characteristics of the isotype heterojunction $n$-GaAs on $n$-Ge diodes. ${ }^{18-25}$

Domains of different sublattice locations are separated by AP boundaries (APBs), which are expected to provide deep levels inside the forbidden band and to act as scattering centers. ${ }^{26,27}$ The problem of the formation of misfit dislocations (MDs), due to the difference in lattice constant between GaAs and $\mathrm{Ge},{ }^{28,29}$ affects solar cell performance, i.e., it decreases in the minority carrier lifetime, increases in the interface recombination velocity, and increases the leakage at the junction, therefore worsening cell performance. ${ }^{30,31}$ One important problem is that $\mathrm{Ge}$ atoms easily evaporate from $\mathrm{Ge}$ substrates during growth and can be incorporated into the epilayer as $n$-type impurities during growth of the solar cells (this is called the autodoping effect ${ }^{32,33}$ ). A high autodoping level may also make a $p$-type layer highly compensated, and this makes it difficult to control the carrier concentration level of an $n$-type layer. This autodoping effect is a serious problem at atmospheric reactor pressure, ${ }^{32,33}$ but was found to be unimportant in low pressure $(20$ mbar) MOVPE reactors. ${ }^{29}$ The main problem thus is to reduce the Ga diffusion into the Ge substrate to avoid the formation of an unwanted $p-n$ junction in the Ge substrate ${ }^{32,33}$ or at the GaAs-Ge heterointerface ${ }^{34}$ that could affect the performance of the GaAs/Ge solar cells. ${ }^{6}$ When $p / n$ GaAs solar cells are grown on $n$-type Ge substrates, an extra photovoltaic (PV) effect is often observed from the current-voltage $(I-V)$ curve of the cells, which implies PV behavior of the $n$-GaAs $/ n$-Ge heterostructure, or less accurately, of the GaAs/Ge interface. This extra PV effect does not always provide extra power 
output and, in fact, it normally reduces the total efficiency of the cell by decreasing its fill factor. The precise mechanism leading to the interface PV effect has not been completely resolved. The two most likely possibilities are variation in the surface states at the GaAs/Ge heterojunction and/or formation of a $p$ - $n$ junction in the Ge substrate from interdiffusion of elements ( $\mathrm{Ga}$ and As into Ge, Ge into GaAs). ${ }^{35} \mathrm{~A}$ detailed study of the optimal growth conditions for APD free GaAs growth on Ge by MOVPE has recently been reported by $\mathrm{Li}$ et $a l .^{36,37}$ They found that a combination of a large substrate offcut toward an in-plane $\langle 110\rangle$ coupled with a high substrate temperature $\left(\sim 650{ }^{\circ} \mathrm{C}\right.$ or higher), relatively low growth rate (below $2 \mu \mathrm{m} / \mathrm{h}$ ), and a high As/Ga ratio $(\sim 60: 1)$ are the requirements for APD free MOVPE GaAs. ${ }^{37}$ No electrical doping measurements were done on these films, but evidence of massive Ge outdiffusion into GaAs grown on $\mathrm{Ge}$ by MOVPE at high growth temperatures and low growth rates has been previously reported. ${ }^{29}$ Since Ge diffusion into GaAs occurs via $\mathrm{Ga}$ vacancies ${ }^{29}$ and the Ga vacancy population will increase with increasing V/III ratio and with decreasing growth rate (i.e., lower Ga flow), it appears that the conditions for APD suppression identified by Li et al. ${ }^{36,37}$ are likely to result in significant Ge outdiffusion, and further work is required to identify MOVPE growth conditions that simultaneously produce APD suppression and chemically sharp interfaces.

Few reports ${ }^{29,38,39}$ on $\mathrm{Si}$-doped GaAs on Ge are available for any indirect information on the interdiffusion phenomena by photoluminescence (PL) investigations. In order to explore the potential application of this material system for devices, such as heterojunction bipolar transistors (HBTs), solar cells, and to study and minimize the effect of interdiffusion of $\mathrm{Ge}, \mathrm{Ga}$, and As atoms across the heterointerface, investigations of the growth, optical, and electrical characteristics of $n$-GaAs $/ n$-Ge heterojunctions grown on Ge substrates were carried out. In this article we report the $I-V$ characteristics of GaAs-Ge heterojunction diodes fabricated by MOVPE in order to gain further understanding of the electron transport mechanisms across the GaAs-Ge $n-n$ isotype heterojunction.

\section{HETEROJUNCTION GROWTH AND PROCESSING}

\section{A. Heterojunction growth}

Undoped and Si-doped $n$-type GaAs were grown in a low pressure horizontal MOVPE reactor on Sb-doped $(\sim 2$ $\left.\times 10^{17} \mathrm{~cm}^{-3}\right) n^{+}$-Ge (100) $6^{\circ}$ misoriented towards the [110] direction. The source materials were trimethylgallium (TMGa), (100\%) arsine $\left(\mathrm{AsH}_{3}\right),(104 \mathrm{ppm})$ silane $\left(\mathrm{SiH}_{4}\right)$ as an $n$-type dopant, and palladium purified $\mathrm{H}_{2}$ as a carrier gas. During growth, the pressure inside the reactor was kept at 100 Torr and the growth temperature was varied from 600 to $725^{\circ} \mathrm{C}$. The TMGa and $\mathrm{AsH}_{3}$ flow rates were varied from 5 to 20 and from 30 to $100 \mathrm{sccm}$, respectively. The total flow rate was kept at about 2 slpm. Prior to growth, the Ge substrates were degreased with organic solvents, then etched in $1 \mathrm{HF}: 1 \mathrm{H}_{2} \mathrm{O}_{2}: 30 \mathrm{H}_{2} \mathrm{O}$ for $15 \mathrm{~s}$ according to specifications given by Ge substrate supplier, Laser Diode Inc.
The double crystal x-ray diffraction (DCXRD) technique was used to measure the perpendicular lattice spacing $a_{\perp}$ of the epitaxial films. PL measurements were carried out using a MIDAC Fourier transform PL (FTPL) system at a temperature of $4.2 \mathrm{~K}$ and $100 \mathrm{~mW}$ laser power for determination of Ge outdiffusion (if any). An argon ion laser operating at a wavelength of $5145 \AA$ was used as a source of excitation. The exposed area was about $3 \mathrm{~mm}^{2}$. The PL signal was detected by a liquid nitrogen $\left(\mathrm{LN}_{2}\right)$ cooled Ge photodetector whose operating range was about $0.75-1.9 \mathrm{eV}$, while the resolution was kept at about $0.5 \mathrm{meV}$. The doping concentrations were determined by a Bio-Rad electrochemical capacitance voltage (ECV) polaron profiler and the capacitance-voltage $(C-V)$ technique. Interdiffusion of $\mathrm{Ge}$, $\mathrm{Ga}$, and As was determined by secondary ion mass spectroscopy (SIMS). $\mathrm{A} \mathrm{Cs}^{+}$ion was used as a primary ion with an acceleration energy of $10 \mathrm{keV}$. The $I-V$ characteristics were determined in order to understand the current transport across the heterointerface.

\section{B. Heterojunction processing for $I-V$ measurements}

The heterojunctions were processed for $I-V$ measurements by making back ohmic contacts using a Au-Ge eutectic with an overlayer of Au. This was accomplished at $\sim 450{ }^{\circ} \mathrm{C}$ for $\sim 2$ min in ultrahigh purity $\mathrm{N}_{2}$ ambient atmosphere. On the as-grown layer, Au dots were made by vacuum evaporation to form Schottky contacts on the top of Si-doped GaAs epitaxial films. In addition, ohmic contacts were also made on top of the epitaxial films and as well as on the back side of the Ge wafer.

Different diode areas were made by using a physical mask that had dots of different sizes. Next, mesas were formed by etching the GaAs using $\mathrm{NH}_{4} \mathrm{OH}: \mathrm{H}_{2} \mathrm{O}_{2}: \mathrm{H}_{2} \mathrm{O}$ (1:1:40) solutions, using the $\mathrm{Au}-\mathrm{Ge}$ dots as masks. The room temperature $I-V$ characteristics of the diodes were checked using an automated arrangement consisting of a Keithley source measuring unit SMU236, an IBM PC486, and a probe station. Since the space quality solar cells work at $300 \mathrm{~K}$ and above, we restrict our discussion to $300 \mathrm{~K}$ only.

\section{RESULTS AND DISCUSSION}

\section{A. Double crystal x-ray diffraction of epi-GaAs on $\mathrm{Ge}$}

The $\mathrm{x}$-ray rocking curves of undoped and Si-doped GaAs/Ge epitaxial layers were examined by DCXRD using $\mathrm{Cu} K \alpha_{1}$ radiation as the x-ray source. Figures 1(a) and 1(b) show typical DCXRD rocking curves from the (400) Bragg lines of the undoped and Si-doped GaAs on Ge substrates; in both cases the epitaxial layer thickness $(\sim 2 \mu \mathrm{m}$ for undoped and $\sim 1 \mu \mathrm{m}$ for Si-doped GaAs epitaxial film) is larger than the critical layer thickness. The average peak separation between the Ge substrate and the undoped and Si-doped GaAs epitaxial layers is about 220 and 200-220 s, respectively. The angular separation, $\Delta \theta$, between the (400) diffraction peaks of $\mathrm{GaAs}$ and $\mathrm{Ge}$ resulting from the difference in lattice plane spacing, $\Delta d / d$, along with their diffraction line pro- 

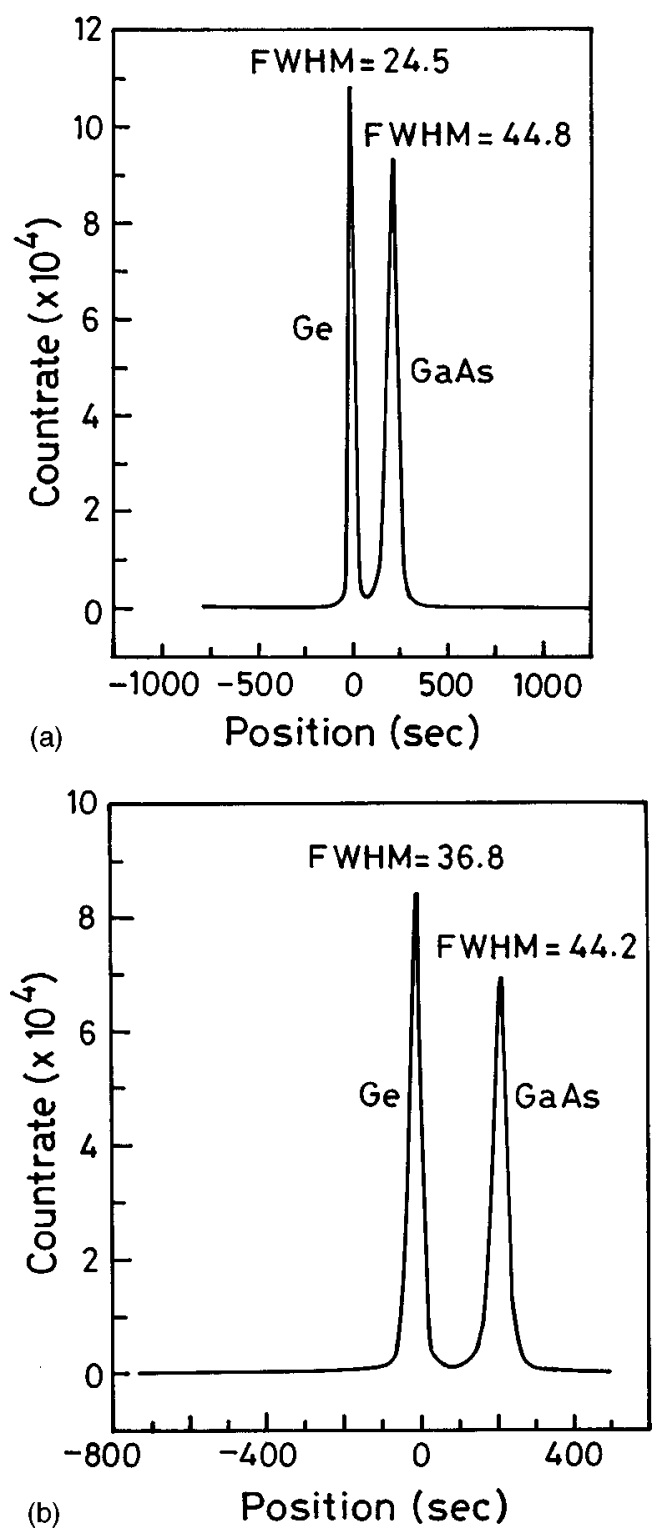

FIG. 1. DCXRD plot of (a) undoped GaAs on Ge and (b) Si-doped GaAs on Ge substrates. The corresponding growth parameters are (a) $T_{s}=625^{\circ} \mathrm{C}$, $[\mathrm{TMGa}]=1.78 \times 10^{-4},\left[\mathrm{AsH}_{3}\right]=1.57 \times 10^{-2}$ and (b) $T_{s}=650^{\circ} \mathrm{C},[\mathrm{TMGa}]$ $=2.67 \times 10^{-4},\left[\mathrm{AsH}_{3}\right]=1.57 \times 10^{-2},\left[\mathrm{SiH}_{4}\right]=5.18 \times 10^{-7}$, respectively.

files provided information about the microstructural quality of the GaAs films. $\Delta \theta$ and the relative lattice mismatch are related by the following equation:

$$
\left(\frac{\Delta a}{a}\right)_{\perp} \equiv \frac{\Delta d}{d} \equiv \frac{-2 \sin (\Delta \theta / 2)}{\tan \theta_{b}},
$$

where $(\Delta a / a)_{\perp} \equiv$ fractional change in the lattice constant, and $\theta_{b}=$ Bragg angle for the (400) plane. The location of the peak associated with the epitaxial layer relative to the substrate, in our case, indicates that the Bragg angle of the epitaxial layer has increased and therefore the lattice has contracted. The deviation of the lattice constant of the GaAs layers on the Ge substrates is calculated from Eq. (1). The deviation of the lattice constant of the GaAs layers on the Ge substrate from that of the bulk is $8.6 \times 10^{-4}$ for undoped
GaAs and $7.6 \times 10^{-4}-8.6 \times 10^{-4}$ for Si-doped GaAs epitaxial film, respectively, after taking into account the anisotropic elastic constants of GaAs. ${ }^{40}$ This shrinkage of the lattice along the depth is due to the elastic deformation that may be caused by stretching of the lattice along the surface because of the difference in thermal shrinking between the GaAs epitaxial layer and the $\mathrm{Ge}$ substrate. The in-plane strain for unrelated epitaxial GaAs on $\mathrm{Ge}$ is $-1.3 \times 10^{-3}$, which would give a perpendicular strain of $+1.9 \times 10^{-3}$ assuming a Poisson ratio of 0.312 , whereas the observed perpendicular strain was $-7.6 \times 10^{-4}$ to $-8.6 \times 10^{-4}$ (lattice contraction). Therefore, the observed in-plane strain is tensile rather than the compressive strain expected from the difference in lattice parameter. Such lattice contractions are normally expected due to the differences in coefficients of thermal expansion between GaAs $\left[5.7 \times 10^{-6}-7.2\right.$ $\times 10^{-6} \mathrm{~K}^{-}$between room temperature and growth temperature $\left.\left(700{ }^{\circ} \mathrm{C}\right)\right]^{41,42}$ and $\mathrm{Ge}\left(5.9 \times 10^{-6}-7.88 \times 10^{-6} \mathrm{~K}^{-1}\right.$ in the same temperature range). ${ }^{43}$ Based upon this, it can be argued that the observed strain arises from the differences in thermal expansion coefficients ${ }^{42}$ and may also be due to some possible strain relaxation generated by misfit dislocations at the interface in the growth temperature regime. ${ }^{42}$ Therefore, the small difference between the lattice constants and thermal expansion coefficients of GaAs and Ge cannot be neglected ${ }^{44}$ and gives rise to the MDs.

\section{B. Low temperature photoluminescence studies}

In order to find out the Ge outdiffusion from the substrates into the GaAs epilayers, low temperature PL (LTPL) spectra were taken on the samples whose DCXRD curves were presented in Fig. 1. Prior to the Si-doped GaAs on Ge growth, undoped GaAs on $\mathrm{Ge}(\sim 2 \mu \mathrm{m})$ was grown in order to check the Ge outdiffusion into the film. Figure 2 shows a PL spectrum of undoped GaAs on the Ge substrate. It can be seen that this film has only one peak at $1.5125 \mathrm{eV}$ corresponding to the acceptor bound exciton with a full width at half maximum (FWHM) of $10.3 \mathrm{meV}$. This PL spectrum suggests the absence of Ge outdiffusion from the substrate, but $\mathrm{Ge}$ outdiffusion was observed by Fischer et al. ${ }^{9}$ and by Masselink et al. ${ }^{45}$ in the molecular beam epitaxy (MBE) growth process and by Timò et al. ${ }^{29}$ in the MOVPE growth process. In our case, the only peak appears at $1.5125 \mathrm{eV}$ for a typical undoped GaAs epitaxial layer on the Ge substrate. If $\mathrm{Ge}$ outdiffusion (the Ge binding energy in GaAs is 43 $\mathrm{meV}$ ) is present inside the GaAs epitaxial films, one could observe the peak at around $1.474 \mathrm{eV}$ at a low temperature PL measurement. Since, there is no peak at around $1.474 \mathrm{eV}$ under our present growth conditions, we can conclude that there is insignificant outdiffusion of Ge inside the GaAs film and hence the film is of high quality. The photoluminescence is indicative of the quality of GaAs epitaxial layers and is not intended to address either the antiphase domain problem or the interface properties.

The above results were presented for the optical quality of the film from the surface. In order to further confirm insignificant outdiffusion of $\mathrm{Ge}$ from the substrate into the GaAs 




FIG. 2. 4.2 K photoluminescence spectra of undoped GaAs on Ge (a) at the surface (solid line) and (b) after etching about $1.3 \mu \mathrm{m}$ (dashed line). The corresponding growth parameters are (a) $T_{s}=625^{\circ} \mathrm{C}, \quad[\mathrm{TMGa}]=1.78$ $\times 10^{-4},\left[\mathrm{AsH}_{3}\right]=1.57 \times 10^{-2}$.

epitaxial film, the PL spectrum was taken after etching the GaAs epitaxial layer through the ECV profiler about $1.3 \mu \mathrm{m}$ from the top surface of the layer. The typical PL spectrum through the ECV etch portion (etch area $\approx 10 \mathrm{~mm}^{2}$ ) of the GaAs epitaxial layer after etching is also shown in Fig. 2. It can be seen that the peak appears at around $1.516 \mathrm{eV}$, which is a free exciton in the GaAs epitaxial film, ${ }^{46}$ and confirms that the film is of high quality. Before etching the film, the PL peak was found at around $1.5125 \mathrm{eV}$, which is an exciton bound to acceptor. The peak at around $1.474 \mathrm{eV}$ was not observed even at the depth of below $1.3 \mu \mathrm{m}$ from the top surface. The small shift $(1.5125 \mathrm{eV})$ in the peak may be attributed to possible Ge outdiffusion into the immediate vicinity of the GaAs film. This results in an increase in carrier concentration and a shift in the PL peak.

The PL spectra of Si-doped GaAs epitaxial films on Ge substrates before and after etching are shown in Fig. 3. The thicknesses of the Si-doped films are around 0.9-1.0 $\mu \mathrm{m}$. It can be seen from Fig. 3 that under the process conditions specified, we did not observe any Ge outdiffusion from the substrate into the epilayer. If Ge outdiffuses (if at all) into the epilayer, the electron concentration should increase because Ge is an $n$-type dopant in GaAs (Ref. 47) under the epitaxial growth conditions used in these experiments. The increase in electron concentration leads to a shift of the PL main peak towards the higher energy side due to the Burstein-Moss effect. ${ }^{48}$ The peak at $1.4864 \mathrm{eV}$ is due to the two-hole transition of an exciton bound to neutral Si donors. ${ }^{49}$ Since the PL main peak energy before and after etching was almost the same (the difference was within the resolution limit), we can conclude that there is insignificant outdiffusion of $\mathrm{Ge}$ into GaAs during growth.

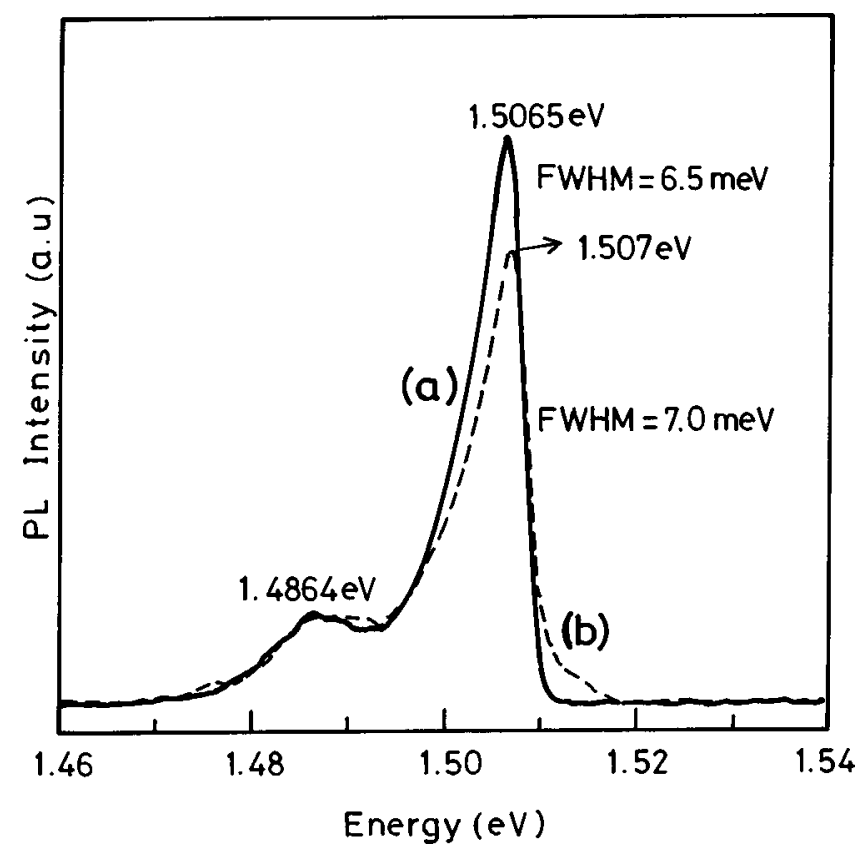

FIG. 3. 4.2 K photoluminescence spectra of Si-doped GaAs on Ge (a) at the surface (solid line) and (b) after etching about $0.8 \mu \mathrm{m}$ (dashed line). The corresponding growth parameters are (a) $T_{s}=625^{\circ} \mathrm{C}, \quad[\mathrm{TMGa}]=1.78$ $\times 10^{-4},\left[\mathrm{AsH}_{3}\right]=1.57 \times 10^{-2},\left[\mathrm{SiH}_{4}\right]=5.18 \times 10^{-7}$.

\section{Secondary ion mass spectroscopy studies}

In order to check the outdiffusion of $\mathrm{Ga}$ and As into Ge and as well as Ge outdiffusion into GaAs films, SIMS was used. It is a powerful technique for quantitative measurements of dopant and impurity levels in semiconductors. The concentration of a particular element can be profiled through a layer using the dynamical SIMS technique. With this method, the mass spectral peak intensity corresponding to a particular ion is monitored as a function of time using a high sputtering rate. Figure 4 shows the depth profiles $(\approx 15 \AA / s)$ of $\mathrm{Ga}, \mathrm{As}, \mathrm{Ge}, \mathrm{C}, \mathrm{Si}$, and $\mathrm{O}$ atoms in the Si-doped $\mathrm{GaAs}$ on Ge, measured by SIMS for a TMGa mole fraction of 2.67 $\times 10^{-4}$. All atoms were barely interdiffused at the heterointerface of the GaAs-Ge $n-n$ system. The abrupt heterointerface in this film indicates approximately $230 \mathrm{~nm}$ outdiffusion of Ge into the GaAs epifilm. In order to check the unwanted $p$ - $n$ junction formation into the Ge substrate by the simultaneous diffusion of $\mathrm{Ga}$ and As, the current-voltage characteristics were obtained on the same epitaxial films, and was shown in the PL spectrum of Fig. 3.

\section{D. $I-V$ characteristics of Schottky contact on Si-doped n-GaAs layer}

Schottky diodes were selected because they are easy to fabricate and study, and very useful information can be obtained from them. The GaAs on Ge diodes was characterized by $C-V$ and $I-V$ techniques to gain further understanding of the conduction dynamics and the extent of Ge diffusion into the GaAs. The electron density was measured by the ECV polaron profiler and further confirmed by conventional $C-V$ measurement. The electron concentrations ranged from 1.0 


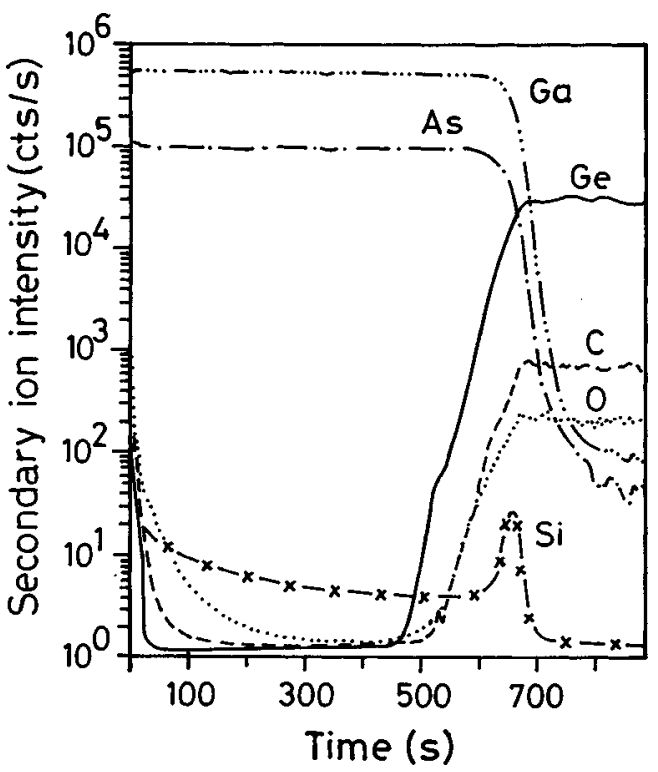

FIG. 4. SIMS depth profiles of compositional atoms around the heterointerface between the Si-doped GaAs epilayer and the Ge (100) substrate (sputtering rate $\approx 15 \AA / \mathrm{s}$ ). The corresponding growth parameters are (a) $T_{s}$ $=650^{\circ} \mathrm{C}, \quad[\mathrm{TMGa}]=2.67 \times 10^{-4}, \quad\left[\mathrm{AsH}_{3}\right]=1.57 \times 10^{-2}, \quad\left[\mathrm{SiH}_{4}\right]=5.18$ $\times 10^{-7}$.

$\times 10^{17}$ to $5 \times 10^{17} \mathrm{~cm}^{-3}$ and are specified in Figs. 5(a) and 5 (b) for better understanding of the conduction process. The typical forward and reverse bias characteristics of the Au- $n$ $\mathrm{GaAs} / n-\mathrm{Ge} / \mathrm{Au}-\mathrm{Ge} / \mathrm{Au}$ Schottky junctions, at different concentrations, are shown in Fig. 5. Using the well known methods discussed in Ref. 50, we measured the diode ideality factor $(n)$ and Schottky barrier heights $\left(\Phi_{\mathrm{Bn}}^{I-V}\right)$, from the forward $I-V$ characteristics. At $300 \mathrm{~K}$ and in the low bias region, the diode ideality factor is rather high and the current is either due to tunneling or generation-recombination current. ${ }^{50}$ For a $p$ - $n$ junction, the modified ideal diode equation $^{51}$ for the $I-V$ characteristics is given by

$$
I \equiv I_{01}\left[\exp \left(\frac{q V}{1 K T}\right)-1\right]+I_{02}\left[\exp \left(\frac{q V}{2 K T}\right)-1\right] .
$$

Equation (2) specifies the current, $I$, for a given applied voltage, $V . I_{01}$ and $I_{02}$ are the reverse saturation currents for the $1 K T$ and $2 K T$ terms, respectively. The $1 K T$ term in Eq. (2) is due to the diffusion of carriers across the junction, assuming that no recombination occurs in the space charge region (SCR); the $2 K T$ term is due to the depletion region generation recombination of carriers in the SCR or at the surface depletion region located around the junction perimeter. For an applied bias greater than a few $K T(\sim 0.1 \mathrm{~V}$ at $300 \mathrm{~K})$ the exponential terms in Eq. (2) dominate and Eq. (2) can therefore be approximated by

$$
I \equiv I_{01} \exp \left(\frac{q V}{1 K T}\right)+I_{02} \exp \left(\frac{q V}{2 K T}\right) .
$$

For a given value of applied bias, $V$, one of the two terms of Eq. (3) will typically be larger than the other. It is seen from Fig. 5 that the $I-V$ characteristics exhibit shunt leakage
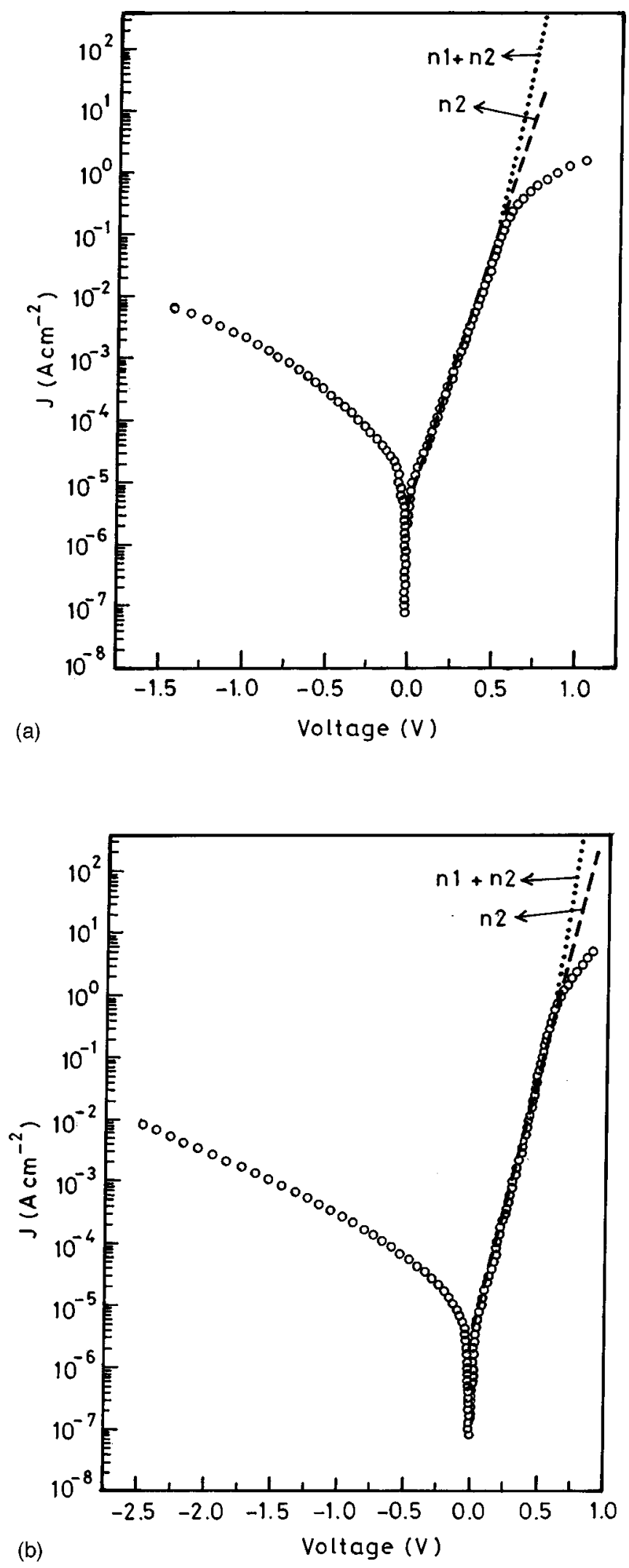

FIG. 5. Current densities vs voltage characteristics of $\mathrm{Au} / n-\mathrm{GaAs} / \mathrm{Ge}$ Schottky diodes. The two different graphs show two different carrier concentrations. The corresponding growth parameters are (a) $T_{s}=625^{\circ} \mathrm{C}$, $[\mathrm{TMGa}]=1.78 \times 10^{-4}, \quad\left[\mathrm{AsH}_{3}\right]=1.57 \times 10^{-2}, \quad\left[\mathrm{SiH}_{4}\right]=5.18 \times 10^{-7}, \quad N_{d}$ $=1.7 \times 10^{17} \mathrm{~cm}^{-3}$ and (b) $T_{s}=650^{\circ} \mathrm{C},[\mathrm{TMGa}]=2.67 \times 10^{-4},\left[\mathrm{AsH}_{3}\right]$ $=1.57 \times 10^{-2},\left[\mathrm{SiH}_{4}\right]=5.18 \times 10^{-7}, N_{d}=2.75 \times 10^{17} \mathrm{~cm}^{-3}$, respectively. 
current at low biases $(<0.2 \mathrm{~V}), 2 K T$ current at moderate $(0.2$ $\mathrm{V}<V<0.5 \mathrm{~V}$ ) biases, and a series resistance effect at high $(>0.5 \mathrm{~V})$ biases. The theoretical Eq. (3) matches excellently with our experimental points. Even the $2 K T$ current component in the theoretical Eq. (3) is also fitted with our experimental points.

From Figs. 5(a) and 5(b), it is seen that the Schottky diodes have soft forward $I-V$ with a lower Schottky barrier height, a large ideality factor, and a larger reverse saturation current. The Schottky diode shown in Fig. 5(a) exhibits a $\Phi_{\mathrm{Bn}}^{I-V}$ of $0.7 \mathrm{eV}, n$ of 1.7 , and a saturation current of $I_{0}=1$ $\times 10^{-6} \mathrm{~A} \mathrm{~cm}^{-2}$. Since the ideality factor is around 2 in three to four decades of current variation, we can conclude that the current is due to either tunneling or generationrecombination current. The soft breakdown voltage and large ideality factor can be explained as follows. The higher ideality factor may be due to either a threading dislocation or due to an APD present inside the film. We did not observe any APD present inside the films within the present set of growth conditions, but we did observe a large number of dislocations inside the films by $A-B$ etch under scanning electron microscopy. ${ }^{52}$ Based on our observation, ${ }^{52}$ we consider the GaAs epitaxial film is free of APDs. This MD may result in the formation of fewer electrical defects. Chand et $a l^{53}$ studied the forward $I-V$ characteristics of GaAs on $\mathrm{Si}$ using a Au-Cr Schottky contact and concluded that the dislocations in $\mathrm{GaAs}$ on $\mathrm{Si}$ act as a conductive path ${ }^{54}$ through the depletion region and such conduction dominates at low reverse bias voltage. They also concluded that, with increasing bias, the current through other mechanisms increases at a faster rate and eventually dominates. Even $\mathrm{GaAs}$ on $\mathrm{Si}$ with higher crystalline quality and lower defect density may not necessarily have better electrical properties if the defects are more electrically active. If we consider the conclusion made by Chand et al. ${ }^{53}$ the dislocations in GaAs on Ge act as electrically active defects which in turn act as a generationrecombination region and hence produces higher ideality factor at lower biases and soft breakdown voltage. On the other hand, the higher ideality factor may be due to the tunneling effect, since the electron concentration inside the film is $\sim 1 \times 10^{17} \mathrm{~cm}^{-3}$ and greater as measured by both the ECV profiler and $C-V$ measurements. The forward characteristics show an ideality factor between $n=1.5$ and 2.14 over nearly three to four orders of magnitude of current densities of different epitaxial films. All the diodes exhibited a low turn-on voltage of $0.5 \mathrm{~V}$ and a saturation level in the range of 1 $\times 10^{-6}-1 \times 10^{-4} \mathrm{~A} \mathrm{~cm}^{-2}$. Under reverse bias the soft breakdown voltage, $V_{\mathrm{br}}$, determined to be the voltage at 10 $\mu \mathrm{A}$, is approximately $3 \mathrm{~V}$.

It is known that the large density of dislocations present in GaAs on Ge substrates is a major problem and it is necessary to understand the electrical activity of the defects and find ways to control them. It is well known that defects can act as generation-recombination centers. They also may getter impurities, causing cross doping near the GaAs/Ge heterointerface. Precipitation or clustering of impurities around the defects may act like random metallic paths, or may generate



FIG. 6. Current densities vs voltage characteristics of $\mathrm{Au} / n-\mathrm{GaAs} / \mathrm{GaAs}$ Schottky diodes. The corresponding growth parameters are $T_{s}=600{ }^{\circ} \mathrm{C}$, $[\mathrm{TMGa}]=1.78 \times 10^{-4}, \quad\left[\mathrm{AsH}_{3}\right]=1.57 \times 10^{-2}, \quad\left[\mathrm{SiH}_{4}\right]=5.18 \times 10^{-7}, \quad N_{d}$ $=1.3 \times 10^{17} \mathrm{~cm}^{-3}$.

local regions of large electric fields, causing a large leakage current and premature breakdown of the device. In addition, the defects may also enhance the tunneling probability of carriers through the depletion region of a device. ${ }^{55}$

On the other hand, the $I-V$ characteristics of Si-doped GaAs on a $n^{+}$-GaAs $\left(2 \times 10^{18} \mathrm{~cm}^{-3}\right)$ substrate is presented in Fig. 6 for comparison and for better understanding of the current conduction mechanism across the GaAs-Ge $n-n$ isotype heterointerface. A similar concentration epitaxial film was chosen and it shows a large $V_{\mathrm{br}}=5 \mathrm{~V}$ at a concentration of $1.3 \times 10^{17} \mathrm{~cm}^{-3}$. The corresponding ideality factor and barrier height for this film are around 1.24 and $0.67 \mathrm{eV}$, respectively. Details of this can be found elsewhere. ${ }^{53,55}$ The $I-V$ characteristics of GaAs on the GaAs substrate were better than those of GaAs on the Ge substrate.

\section{E. I-V characteristics of Si-doped $n$-GaAs $/ n$-Ge heterostructure with ohmic contacts}

To investigate whether a $p$ - $n$ junction is formed inside Ge substrates, mesa diodes were formed by etching GaAs with $\mathrm{NH}_{4} \mathrm{OH}: \mathrm{H}_{2} \mathrm{O}_{2}: \mathrm{H}_{2} \mathrm{O}$ (1:1:40) with a Au-Ge eutectic alloy as the ohmic contact. Figure 7 compares the $I-V$ characteristics of GaAs on Ge diodes that have various diode areas. The $I-V$ characteristics show a near linear behavior/ohmic characteristics over a wide range of applied voltages. This result could not be explained by $p$ - $n$ junction formation inside Ge substrates during MOVPE growth of GaAs. The absence of such $p$ - $n$ junction formation inside the Ge substrates was also confirmed by a Bio-Rad ECV polaron profiler. Figure 8 shows one of the ECV carrier concentration profiles of Si-doped GaAs on the Ge substrate. From Fig. 8 it is also seen that the 


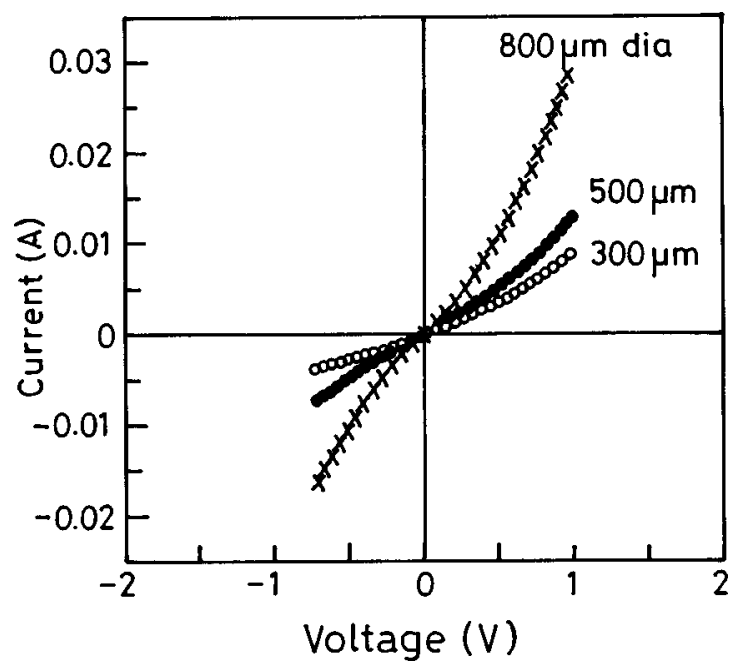

FIG. 7. Current densities vs voltage characteristics of $\mathrm{Au}-\mathrm{Ge} / n-\mathrm{GaAs} / \mathrm{Ge}$ mesa diodes. The corresponding growth parameters are $T_{s}=725^{\circ} \mathrm{C}$, $[\mathrm{TMGa}]=1.78 \times 10^{-4},\left[\mathrm{AsH}_{3}\right]=1.57 \times 10^{-2},\left[\mathrm{SiH}_{4}\right]=1.24 \times 10^{-8}, N_{d}=5$ $\times 10^{17} \mathrm{~cm}^{-3}$.

concentration is uniform along the depth and there are almost sharp interfaces between the Si-doped GaAs and the Ge substrate. The resolution was kept at around $0.05 \mu \mathrm{m}$ during the etching process. It can also be seen from Fig. 8 that the carrier concentration increases inside the Ge substrate due to As diffusion during the MOVPE growth process. Wojtczuk et al. ${ }^{11,56}$ reported in their results of GaAs on Ge substrates that the conduction mechanism is dominated by a $p-n$ junction formed inside the $n$-Ge substrates, either the Si-doped



FIG. 8. Electrochemical $C-V$ profile of Si-doped GaAs on the Ge substrate. The corresponding growth parameters are $T_{s}=675^{\circ} \mathrm{C},[\mathrm{TMGa}]=1.78$ $\times 10^{-4},\left[\mathrm{AsH}_{3}\right]=1.57 \times 10^{-2},\left[\mathrm{SiH}_{4}\right]=1.24 \times 10^{-9}$.
GaAs or the Zn-doped GaAs, grown by the MOVPE process. The rectification properties are controlled by the presence of a $p$ - $n$ junction at or inside the Ge substrates in their results. From Fig. 7, it is seen that the $I-V$ characteristics indicate an ohmic conduction not controlled by $p-n$ junction formation ${ }^{5}$ inside the Ge substrates. It is also seen from Fig. 7 that the current is higher in larger diode areas. One can determine that the current versus diameter of the diode was not linear for a fixed value of applied voltage. This means that there was no side leakage in the mesa diodes.

\section{CONCLUSIONS}

Undoped and Si-doped GaAs epitaxial films on Ge substrates grown by MOVPE were characterized by DCXRD, LTPL, SIMS, ECV profiler, and electrical transport across the $n$-GaAs $/ n$-Ge heterointerface. A plethora of growth conditions appear in the literature concerning the attempt to grow APD-free GaAs on Ge. In the present case, even though the growth temperature regime is close to the reported values, the main difference in minimizing APD formation may arise from the growth rates $(\sim 3 \mu \mathrm{m} / \mathrm{h})$ and the $\mathrm{V} / \mathrm{III}$ ratio $(\sim 88)$. The studies indicated little Ge outdiffusion, as was envisaged by a small shift in the PL peak and may be insufficient to influence the carrier density more than 200-300 nm from the interface. Ge related peaks were not observed even after etching the Si-doped GaAs epitaxial films from the surface of about $0.8 \mu \mathrm{m}$. The carrier concentration was found to be the same along the depth of the film. The secondary ion mass spectroscopy results showed that all the atoms barely interdiffused across the GaAs/Ge heterointerface and resulted in no $p-n$ junction formation inside the Ge substrate. This result indicates approximately $230 \mathrm{~nm}$ of Ge outdiffusion into the GaAs epitaxial film during MOVPE growth of GaAs on the Ge substrate. The $I-V$ characteristics of Si-doped GaAs on Ge substrates using Au Schottky diodes show a higher ideality factor, lower barrier height, and soft breakdown voltage. The higher ideality factor may be due to either tunneling or generation-recombination current and the lower breakdown may be due to the dislocations present inside the GaAs films which in turn produce electrically active defects and hence produce a higher ideality factor at lower biases. The GaAs/Ge heterointerface does not show any rectification properties after making ohmic contacts on both sides followed by mesa etching. This indicates that there was no $p$ - $n$ junction formation at or near the heterointerface of GaAs/Ge and this was further confirmed by the ECV carrier concentration profile. The passive nature of the GaAs/Ge heterointerface is an encouraging step towards the development of space quality GaAs/Ge solar cells.

\section{ACKNOWLEDGMENTS}

The authors wish to thank Dr. K. S. R. K. Rao for providing PL measurement facilities, Professor D. N. Bose, Indian Institute of Technology, Kharagpur, for providing DCXRD facilities, and R. Ashwatraman, Indira Ghandhi Center for Atomic Research, Kalpakkam, for SIMS analysis. 
${ }^{1}$ R. A. Metzger, Compd. Semicond. 2, 25 (1996).

${ }^{2}$ M. Kato, K. Mitsui, K. Mizuguchi, N. Hayafuji, S. Ochi, Y. Yukimoto, T. Murotani, and K. Fujikawa, Proceedings of the 18th IEEE Photovoltaic Specialists Conference (IEEE, New York, 1985), p. 14.

${ }^{3}$ C. Flores, D. Passoni, and G. Timò, Proceedings of the European Space Power Conference, Spain, ESA SP-294, 1989, p. 507.

${ }^{4}$ C. Flores, B. Bollani, R. Campesato, F. Paletta, G. Timò, and A. Tosoni, Sol. Energy Mater. 23, 356 (1991).

${ }^{5}$ K. I. Chang, Y. C. M. Yeh, P. A. Iles, J. M. Tracy, and R. K. Morris, Proceedings of the 19th IEEE Photovoltaic Specialists Conference (IEEE, New York, 1987), p. 273.

${ }^{6}$ P. A. Iles, Y. C. M. Yeh, F. H. Ho, C. L. Chu, and C. Cheng, IEEE Electron Device Lett. EDL-11, 140 (1990).

${ }^{7}$ N. Chand, J. Klem, and H. Morkoç, Appl. Phys. Lett. 48, 484 (1986).

${ }^{8}$ S. C. Martin, L. M. Hitt, and J. J. Rosenberg, IEEE Electron Device Lett. EDL-10, 325 (1989).

${ }^{9}$ R. Fischer, W. T. Masselink, J. Klem, T. Henderson, T. C. McGlinn, M. V. Klein, H. Morkoç, J. H. Mazur, and J. Washburn, J. Appl. Phys. 58, 374 (1985).

${ }^{10}$ C. Flores, B. Bollani, R. Campensato, D. Passoni, and G. L. Timò, Microelectron. Eng. 18, 175 (1992).

${ }^{11}$ S. J. Wojtczuk, S. P. Tobin, C. J. Keavney, C. Bajgar, M. M. Sanfacon, L. M. Geoffroy, T. M. Dixon, S. M. Vernon, J. D. Scofield, and D. S. Ruby, IEEE Trans. Electron Devices ED-37, 455 (1990).

${ }^{12}$ S. Strite, D. Biswas, N. S. Kumar, M. Fradkin, and H. Morkoç, Appl. Phys. Lett. 56, 244 (1990).

${ }^{13}$ P. R. Pukite and P. I. Cohen, J. Cryst. Growth 81, 214 (1987).

${ }^{14}$ S. Strite, M. S. Unlu, K. Adomi, G.-B. Gao, A. Agarwal, A. Rockett, H. Morkoç, D. Li, Y. Nakamura, and N. Otsuka, J. Vac. Sci. Technol. B 8, 1131 (1990).

${ }^{15}$ S. Strite, M. S. Unlu, A. L. Demirel, D. S. L. Mui, and H. Morkoç, J. Vac. Sci. Technol. B 10, 675 (1992).

${ }^{16}$ R. S. Bauer and J. C. Mikkelson, J. Vac. Sci. Technol. 21, 491 (1982).

${ }^{17}$ J. H. Neave, P. K. Larsen, B. A. Joyce, J. P. Gowers, and J. F. Van der Veen, J. Vac. Sci. Technol. B 1, 668 (1983).

${ }^{18}$ J. M. Ballingall, R. A. Stall, C. E. C. Wood, and L. F. Eastman, J. Appl. Phys. 52, 4098 (1981).

${ }^{19}$ J. M. Ballingall, C. E. C. Wood, and L. F. Eastman, J. Vac. Sci. Technol. B 1, 675 (1983).

${ }^{20}$ J. C. De Jaeger and G. Salmer, IEE Proc., Part I: Solid-State Electron Devices 127, 207 (1980).

${ }^{21}$ F. C. Jain and M. A. Melehy, Appl. Phys. Lett. 27, 36 (1975).

${ }^{22}$ R. L. Anderson, Solid-State Electron. 5, 341 (1962).

${ }^{23}$ L. L. Chang, Solid-State Electron. 8, 721 (1965).

${ }^{24}$ D. S. Howarth and D. L. Feucht, Appl. Phys. Lett. 23, 365 (1973).

${ }^{25}$ H. Kroemer, W. Y. Chen, J. S. Jarris, Jr., and D. D. Edwall, Appl. Phys. Lett. 36, 295 (1980).

${ }^{26}$ H. Kroemer, J. Cryst. Growth 81, 193 (1987).

${ }^{27}$ P. M. Petroff, J. Vac. Sci. Technol. B 4, 874 (1986)

${ }^{28}$ L. Lazzarini, Y. Li, P. Franzosi, L. J. Giling, L. Nasi, F. Longo, M. Urchulutegui, and G. Salviati, Mater. Sci. Eng., B 28, 502 (1992).

${ }^{29}$ G. Timò, C. Flores, B. Bollani, D. Passoni, C. Bocchi, P. Franzosi, L. Lazzarini, and G. Salviati, J. Cryst. Growth 125, 440 (1992).
${ }^{30}$ J. C. Zolper and A. M. Baruet, Proceedings of the 20th IEEE Photovoltaic Specialists Conference (IEEE, New York, 1988), p. 678.

${ }^{31}$ M. A. Green, Solar Cells, Operating Principles, Technology and System Applications (Prentice-Hall, Englewood Cliffs, NJ, 1982).

${ }^{32}$ S. P. Tobin, S. M. Vernon, C. Bajgar, V. E. Haven, Jr., and S. E. Davis, in Ref. 2, p. 134.

${ }^{33}$ S. P. Tobin, S. M. Vernon, C. Bajgar, V. E. Haven, Jr., L. M. Geoffroy, M. M. Sanfacon, D. R. Lillington, R. E. Hart, K. A. Emery, and R. J. Matson, in Ref. 30, p. 405.

${ }^{34}$ L. D. Partain, G. F. Virshuf, and N. R. Kaminar, in Ref. 30, p. 759.

${ }^{35}$ Y. C. M. Yeh, K. I. Chang, C. H. Cheng, F. Ho, and P. Illes, in Ref. 30, p. 451.

${ }^{36}$ Y. Li, L. Lazzarini, L. J. Giling, and G. Salviati, J. Appl. Phys. 76, 5748 (1994).

${ }^{37}$ Y. Li, G. Salviati, M. M. G. Bongers, L. Lazzarini, L. Nasi, and L. J. Giling, J. Cryst. Growth 163, 195 (1996).

${ }^{38}$ G. Timò, L. Solevi, and T. H. Nhung, Mater. Sci. Eng., B 28, 474 (1994).

${ }^{39}$ G. L. Timò and C. Flores, Proceedings of the 1st IEEE World Conference Photovoltaic Energy Conversion, Waikoloa, Hawaii, 1994, p. 2000.

${ }^{40}$ R. Venkatasubramanian, K. Patel, and S. K. Ghandhi, J. Cryst. Growth 94, 34 (1989).

${ }^{41}$ Encyclopedia of Applied Physics, edited by G. L. Trigg (VCH, Wertheim, 1993), Vol. 7, p. 43.

${ }^{42}$ T. B. Light, M. Berkenblit, and A. Reisman, J. Electrochem. Soc. 115, 969 (1969).

${ }^{43}$ G. A. Slack and S. F. Bartram, J. Appl. Phys. 46, 89 (1975).

${ }^{44}$ D. Eres, D. H. Lowndes, J. Z. Tischler, J. W. Sharp, T. E. Haynes, and M. F. Chisholm, J. Appl. Phys. 67, 1361 (1990).

${ }^{45}$ W. T. Masselink, R. Fischer, J. Klem, T. Henderson, P. Pearah, and H. Morkoç, Appl. Phys. Lett. 45, 457 (1984).

${ }^{46}$ M. K. Hudait, P. Modak, S. Hardikar, and S. B. Krupanidhi, Solid State Commun. 103, 411 (1997).

${ }^{47}$ S. J. Bass, J. Cryst. Growth 47, 613 (1979).

${ }^{48}$ E. Burstein, Phys. Rev. 93, 632 (1954); T. S. Moss, Proc. Phys. Soc. London, Sect. B 67, 775 (1954).

${ }^{49}$ L. Pavesi and M. Guzzi, J. Appl. Phys. 75, 4779 (1994).

${ }^{50}$ E. H. Rhoderick and R. H. Williams, Metal-Semiconductor Contacts (Clarendon, Oxford, 1988).

${ }^{51}$ G. W. Neudeck, Modular Series on Solid State Devices, Vol. II, The PN Junction Diode (Addison-Wesley, Reading, MA, 1983), p. 69.

${ }^{52}$ P. Modak, M. K. Hudait, S. Hardikar, and S. B. Krupanidhi, J. Cryst. Growth 193, 501 (1998).

${ }^{53}$ N. Chand, F. Ren, A. T. Macrander, J. P. Van der Ziel, A. M. Sergent, R. Hull, S. N. G. Chu, Y. K. Chen, and D. V. Lang, J. Appl. Phys. 67, 2343 (1990).

${ }^{54}$ W. Shockley, Solid State Technol. 26, 75 (1983).

${ }^{55}$ N. Chand, R. Fischer, A. M. Sergent, D. V. Lang, S. J. Pearton, and A. Y. Cho, Appl. Phys. Lett. 51, 1013 (1987).

${ }^{56}$ S. J. Wojtczuk, S. P. Tobin, M. M. Sanfacon, V. E. Haven, L. M. Geoffroy, and S. M. Vernon, Proceedings of the 22nd IEEE Photovoltaic Specialists Conference (IEEE, New York, 1991), p. 73. 